\title{
Filigrane
}

Écoutes psychanalytiques

\section{Aux origines de l'éthique ? Le féminin-maternel chez tout humain. Entretien avec Viviane Chetrit-Vatine}

\section{Élie Battat}

Volume 24, numéro 1, printemps 2015

URI : https://id.erudit.org/iderudit/1033083ar

DOI : https://doi.org/10.7202/1033083ar

Aller au sommaire du numéro

Éditeur(s)

Revue Santé mentale au Québec

ISSN

1192-1412 (imprimé)

1911-4656 (numérique)

Découvrir la revue

Citer ce document

Battat, É. (2015). Aux origines de l'éthique ? Le féminin-maternel chez tout humain. Entretien avec Viviane Chetrit-Vatine. Filigrane, 24(1), 55-72.

https://doi.org/10.7202/1033083ar d'utilisation que vous pouvez consulter en ligne. 


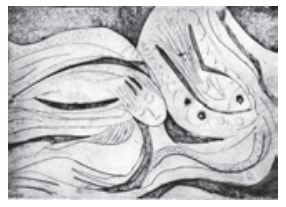

\section{Aux origines de l'éthique? Le féminin- maternel chez tout humain. Entretien avec Viviane Chetrit-Vatine ${ }^{1}$}

\section{Élie Battat}

Élie Battat: Bonjour docteure Viviane Chetrit-Vatine. Je suis très heureux de vous rencontrer ici à Montréal et très heureux que vous ayez accepté notre proposition de faire cette entrevue pour la revue Filigrane. Docteure Chetrit-Vatine, vous êtes d'Israël, vous êtes psychanalyste, vous êtes membre de la Société psychanalytique d'Israël, membre de l'Institut psychanalytique d'Israël, vous faites de la formation, de l'enseignement, et du travail clinique à Jérusalem et à Tel Aviv. Vous êtes également chercheure associée au CRPMS, le Centre de recherche psychanalyse médecine et société à l'Université Diderot (Paris). Vous êtes auteure de plusieurs articles, entre autres dans la Revue française de psychanalyse, et vous êtes également auteure d'un livre paru en 2012 aux Presses universitaires de France: La séduction éthique de la situation analytique; aux origines féminines maternelles de la responsabilité pour l'autre ${ }^{2}$. Nous vous invitons d'ailleurs à nous en parler aujourd'hui.

Voilà un titre qui parle de séduction éthique, de responsabilité pour l'autre et de ses origines féminines maternelles: un titre qui annonce déjà une très grande richesse de la pensée au sujet de la situation analytique. Un livre qui nous interpelle, car il nous plonge au cœur de la rencontre interhumaine dans la pratique de la psychanalyse. À cet effet, vous soulevez une question fondamentale et je vous cite: "comment la psychanalyse peut-elle encore se permettre de ne pas donner toute sa place à la dimension de la rencontre inter-humaine, et ne pas prendre en compte sérieusement que c'est bien au cœur de cette rencontre qu'elle se pratique?». Pour débuter notre entretien, pourriez-vous nous parler de votre parcours: nous dire comment vous en êtes arrivée à faire ce travail qui est d'une richesse colossale, et qui m’a beaucoup interpellé, tant il résonne sur le plan du travail clinique? 
Viviane Chetrit-Vatine: Dans les années 1990-1991, on a créé à Jérusalem une Maison Verte. C'est un projet qui fut originairement conçu à Paris par Françoise Dolto; elle a toujours mis l'accent sur la relation à l'autre comme relation à un sujet - il est vrai qu'elle était d'inspiration lacanienne. Nous avons donc créé ce cadre où l'on rencontrait de tout petits enfants accompagnés de parents ou de tout adulte s'occupant d'eux; il s'agissait pour nous d'être présents et de s'adresser à eux comme sujets. Je parle ici de tout petits bébés et de leurs parents, ou des personnes qui les accompagnaient. Pour moi, depuis très longtemps, la question de la relation psychanalytique investie dans une relation inter-humaine a été quelque chose de très prégnant. Françoise Dolto parlait d'ailleurs de psychanalyse de cette manière-là. Du reste, cet intérêt est évidemment relié à mon histoire personnelle, quelque chose qui a été très tôt présent chez moi.

La première fois que j'ai approché la psychanalyse, j'avais quatre ans et demi... Parce que j'étais devenue agressive envers mon petit frère, alors âgé d'un an, mes parents m'envoyèrent chez un couple de psychothérapeutes qui avaient apparemment une perspective psychanalytique. La troisième séance est restée gravée en moi: je me souviens d'une petite poupée qu'il fallait mettre dans une baignoire. L'eau a commencé à couler parce que je rentrais le bébé un petit peu trop profondément dans la baignoire. L'eau coulait sur un parquet verni; j'étais fort inquiète, car à la maison, si l'eau s'était mise à couler sur le parquet... J'ai relevé la tête et, tel qu'inscrit dans ma mémoire, j'ai vu le visage de cette femme, la thérapeute, qui au même moment me regardait avec un sourire merveilleux. Mes parents, peu au fait de la teneur d'une rencontre analytique, considérèrent ce couple comme complètement fou: tout cela n'avait évidemment rien à voir avec le complément d'éducation que j'étais, selon eux, censée recevoir. Ils m'enlevèrent de là sans plus attendre. Il n'y eut donc que trois séances.

Comme dans l'histoire des fraises convoitées mais interdites, dont il ne reste plus qu'à rêver, cette expérience laissa ses traces, indélébiles. Une inscription ensoleillée faite de ce sourire, de cette offre: exprimer simplement une fraiche pulsionnalité et la sentir accueillie... C'est pour moi la première image d'une relation qui recèle de l'inter-humain à proprement parler: accueil et signification. Car il m'était apparu clairement, toute petite fille que j'étais, que ce que j'exprimais faisait sens.

ÉB: Vous avez connu le cour de la rencontre inter-humaine à ce moment-là. VCV: Exactement! Bon après, pour ce qui est de la question de la séduction, de la responsabilité, cela est en lien avec mon père qui était quelqu'un 
à la fois de fort séduisant, et en même temps doué d'une position éthique évidente qui passait par la responsabilité pour l'autre. C'était quelqu'un d'engagé, qui a été pour moi une figure d'identification très importante. J'ai réalisé après sa mort (il est mort en 1992), que cette position de responsabilité très forte dont il parlait si souvent avait trouvé sa source dans une identification à sa mère. C'est ainsi que s'est précisée en moi l'idée d'une origine féminine maternelle de cette capacité humaine qu'est la faculté de responsabilité pour l'autre, qu'il s'agisse de sujets masculins ou féminins, d'hommes ou de femmes. J'ai donc réalisé que cette capacité, mon père se l'était appropriée par identification à sa mère, qui elle, lui avait transmis ces valeurs. Mon père était aussi quelqu'un qui, d'une façon manifeste, incarnait pour mes frères et moi-même, la loi. En vérité, ce qui a été transmis n'est pas de l'ordre de la loi, mais bien de l'ordre de l'éthique comme responsabilité, comme rencontre. Tout en travaillant la thèse en question, je savais que séduction et éthique avaient rapport avec lui, mais aussi avec les origines féminines maternelles de cette capacité. J'ai réalisé combien cette élaboration théorique avait nécessité une prise de conscience de ce qui avait, chez mon père, motivé une telle capacité.

ÉB: Alors, la séduction éthique, pouvez-vous nous dire comment vous la voyez s'articuler dans la situation analytique?

VCV: Oui... En fait, la séduction éthique se joue dans la rencontre analytique, en particulier au moment de l'offre d'analyse. Puis, on peut la voir à l'oeuvre au cours du processus lui-même, en particulier dans des moments d'impasse, de même que dans tout ce qui se déploie autour du cadre.

Dans la troisième partie de mon livre, $j$ 'ai proposé un certain nombre d'exemples cliniques où l'on voit comment, par exemple, l'offre d'analyse est en fait l'offre d'un analyste: c'est une offre humanisée. Cette offre est nécessairement vécue par l'analysant potentiel comme une séduction, au sens où on lui demande de dire tout ce qu'il a en tête, au sens où on lui offre la possibilité d'être dans une relation très intime, dès le départ, avec quelqu'un de tout à fait étranger. C'est en même temps quelque chose qui est extrêmement captivant: qui n'en voudrait pas de ce type de relation? D’un autre côté, chez des patients qui ont été traumatisés ou séduits d'une manière traumatique, cette offre peut être vécue comme une réédition de ce traumatisme, et une position paranoïde peut alors s'exprimer.

En tous les cas, cette dimension de séduction apparaît au moment de l'offre d'analyse. Je pense, par exemple, aux analystes en formation qui recherchent des patients susceptibles d'être intéressés à faire une analyse. Ils 
peuvent proposer éventuellement à tel ou tel de leur patient en psychothérapie de passer à une analyse, mais ce saut, qu'est-ce qu'il représente? Qu'est-ce qu'ils transmettent à leurs patients, et qu'est-ce qui se passe à ce moment-là? Chez certains patients cela déclenche effectivement une position analytique, une capacité d'analyser; chez d'autres, cela crée des troubles terribles. Je pense que l'important, en particulier au moment de l'offre d'analyse, est de réaliser l'aspect séducteur de cette offre et le fait que l'analyste incite le transfert.

D'ailleurs, Laplanche exprime très clairement que le transfert est incité par l'analyste lui-même et par la situation analytique. Ce que j'ai essayé d'apporter par rapport à Laplanche, c'est qu'au moment de la naissance, l'enfant rencontre un monde adulte doué de sexualité adulte et traversé par un inconscient par définition sexuel (selon Laplanche), mais il rencontre aussi, simultanément, un monde adulte mobilisé au niveau de sa responsabilité par rapport à lui.

Parallèlement, dans la situation analytique, un transfert de séduction originaire se réactualise, en même temps qu'un «transfert d'espace matriciel» se met en place. Je veux dire par là, un transfert où le patient attend que quelqu'un - l'analyste en l'occurrence — prenne une responsabilité entière par rapport à lui. Cette attente, de la part des analysants, qu'on s'occupe d'eux, qu'on les prenne en charge, etc., paraît parfois régressive, donc il ne serait pas question d'y répondre. Or, ma position est que l'analyste peut être interpellé par ce qui s'exprime à ce moment-là, par ce qui est évoqué. Cette interpellation, je l'appelle «saisissement». Il y a un saisissement éthique qui en principe, se passe chez un analyste dans la rencontre avec un nouveau patient, ce qui suscite et incite ce transfert d'espace matriciel. Ce transfert n’est pas nécessairement quelque chose qui s'est passé au départ de la vie de cette même personne, mais l'enfant, en principe, vient au monde avec cette attente-là. Freud avait évoqué l' «attente croyante». En vérité, il y a quelque chose dans cette " attente croyante » qui correspond, à mon avis, à une attente relative à l'auto-conservation, une exigence éthique de la part des adultes qui vont prendre en charge un enfant. Cette attente, cette exigence, se réactualise quand il y a offre d'analyse. L'idée de séduction éthique, en tant que l'éthique réfère à la responsabilité pour l'autre, est impliquée dans la situation analytique dont l'analyste fait partie intégrante. On ne peut parler de situation analytique sans parler de l'implication nécessaire de l'analyste à l'intérieur de cette situation.

ÉB: Vous distinguez trois types de transfert: le transfert classique, le transfert de séduction originaire, et vous introduisez le transfert d'espace 
matriciel. Vous dites que dans le transfert d'espace matriciel, le psychanalyste est interpellé par l'état de désaide du patient ou de l'enfant et vous comparez cela à la situation de séduction originaire chez Laplanche. Le psychanalyste est alors renvoyé à sa propre expérience de désaide. Pouvez-vous nous en parler?

VCV : Effectivement, dans mon livre, cela est présenté ainsi. À la réflexion, je requestionne cette position. Monique Schneider a écrit un livre qui s'appelle Aux origines de l'éthique, la détresse, où elle aborde le désaide en question. Mais Levinas considère que cette interpellation du sujet éthique par l'autre, par «le visage» de l'autre, est une interpellation qui le trouve en situation de bien-être, et le dérange dans son bien-être. En ce sens, je ne suis pas convaincue que ce qui nous active est nécessairement l'identification avec la détresse ou le désaide. Il y a quelque chose dans la rencontre à l'autre qui nous mobilise; l'aspect détresse de cet autre est une partie peut-être de cette mobilisation, mais ce n'est pas tout. Il y a dans la rencontre à l'autre, avec l'humain, la rencontre avec quelque chose qui nous dépasse, qui nous interpelle et qui nous déstabilise dans notre bien-être. En ce sens, je ne voudrais pas réduire la mobilisation - de l'analyste et du sujet éthique — à une identification ou à une empathie à sa détresse. Ce n'est pas que de cela qu'il s'agit.

Hans Jonas a écrit un ouvrage intitulé Le principe responsabilité. Dans un chapitre, auquel je me réfère, il dit que l'on retrouve la situation de responsabilité primordiale dans la rencontre avec le nouveau-né. De fait, personne ne peut passer devant un nouveau-né abandonné sans être traversé par un élan vers celui-ci afin de s'occuper de lui, sans se sentir interpellé et amené à en prendre la responsabilité. Est-ce parce qu'il voit l'enfant en détresse, ou est-ce qu'il y a quelque chose, dans cette humanité tout à fait première, qui a besoin de l'autre, qui le mobilise, et qui vient le déstabiliser dans sa tranquillité? Je ne suis pas complètement convaincue de l'idée que ce qui nous mobilise, c'est la détresse de nos patients; c'est en partie vrai mais ce n'est pas que cela. Il y a quelque chose dans l'étrangeté de la rencontre avec un nouveau patient, et en quelque sorte à chaque nouvelle session, qui nous déstabilise dans notre tranquillité et qui nous saisit quelque part. Ensuite, rapidement, un processus de transformation s'opère, que j'appelle «transformation en position matricielle»; à partir de ce moment, nous sommes en position d'écoute, d'accueil et de responsabilité par rapport au patient. Je parle de quelque chose qui réfère à la détresse, mais pas que de ça. Quelque chose qui nous dépasserait en tant que rencontre du visage de l'autre, de cet autre qui est autre que moi-même et qui est humain. 
ÉB: Dans votre livre, vous parlez de la passion de l'analyste, et vous l'associez à la passion maternelle.

VCV: Comme je l'ai écrit, la rencontre entre Laplanche et Levinas n'est pas une rencontre consensuelle. S'ils s'étaient rencontrés ou s'ils avaient lu ce que j'ai écrit, il y a de fortes chances qu'ils auraient largement critiqué telle ou telle proposition. Par exemple en ce qui concerne la question de l'affect de l'analyste, je parle en termes de responsabilité affectée, et ce n'est pas du tout la direction de Laplanche. Il est important d'insister là-dessus parce qu'aujourd'hui, les patients qui demandent à être en cure analytique ont en général des problèmes au niveau de leur identité, au niveau de l'«axe intérieur» qui leur permet de fonctionner et de vivre. Nous avons tous le même genre de patients aujourd'hui: des problématiques narcissiques, borderlines... Pour que quelque chose puisse se passer, une présence affectée est nécessaire de la part de l'analyste. C'est-à-dire que ce sont des personnes qui très souvent ont manqué justement d'une expérience de cet ordre. Ces patients ont parfois eu des parents trop aimants, parce que trop narcissiques et incapables de voir suffisamment l'enfant pour là où il était; dans ce trop, il y a quelque chose qu'on doit arriver à défaire, à assouplir, à adoucir, à diluer. Ou encore, ces patients ont manqué de cet amour parental. Cela revient au même: qu'il y ait du trop ou du manque, il y a toujours du trop à l'intérieur. Paradoxalement, pour que quelque chose puisse se passer, il faut que l'analyste soit affecté.

Si l'analyste est en situation de neutralité froide, cela risque de susciter chez le patient un vécu de re-traumatisation: à nouveau, il y aura un effet de trop et d'excès qui peut provoquer soit une rage justifiée, soit un départ prématuré. Il y a des tas de gens aujourd'hui qui ne peuvent pas tenir en analyse avec des thérapeutes de ce genre. Personnellement, je suis dans la lignée de René Roussillon qui parle de l'importance de l'affect pour permettre un processus de symbolisation et un processus d'appropriation subjective. C'est un aspect qui a été longtemps négligé. Jean-Luc Donnet, un psychanalyste fort connu qui a beaucoup travaillé la question de la situation analytique, la situation analysante, le divan tempéré, etc., parle aujourd'hui en termes de neutralité engagée. On y retrouve à peu près la même idée: moi j’insiste sur la responsabilité asymétrique et affectée.

Cela vaudrait la peine de parler un petit peu de l'asymétrie. Le départ de ma réflexion, au niveau plus théorique (au-delà de toutes les motivations plus personnelles dont j'ai pu parler auparavant), était ma préoccupation de trouver un sous-bassement théorique au fait que nous sommes, en tant qu'analystes, dans une relation asymétrique par rapport à nos patients. Une 
situation qui a longtemps pu être pensée en termes de pouvoir. L'école intersubjective et relationnelle américaine a commencé à discuter abondamment de cet aspect pour des raisons notamment politiques. Qu'est-ce que cette asymétrie? Que sont ces effets de pouvoir? J'étais profondément convaincue de l'importance de cette asymétrie pour qu'un processus puisse s'établir, se développer. Mais en même temps, comment le fonder théoriquement, non pas dans un ordre de pouvoir, mais dans un autre ordre? Alors que j'étais en formation psychanalytique, j'ai commencé à m’intéresser à Levinas qui insiste énormément sur l'aspect asymétrique de cette responsabilité pour l'autre. Quand je suis arrivée en fin de cursus, j'ai commenté justement cet aspect-là à partir d'un travail avec une petite fille, dont je parle d'ailleurs dans mon livre: je m'étais rendu compte que quelque chose s'était passé, qui avait permis chez elle une transformation significative du fait d'interventions de ma part qu'elle avait vécues comme relevant justement de cette asymétrie responsable par rapport à elle. C'était là un premier élément théorique possible afin de soutenir la nécessité de cette asymétrie. Et puis, pendant l'été 1998, nous avons eu une rencontre en Israël avec des analystes de plusieurs sociétés européennes, rencontre organisée en collaboration avec la Fédération européenne de psychanalyse. Nous y avons discuté du Babel des langues en psychanalyse. À un moment donné, j'ai cité Levinas, qui m’interpellait déjà en lien avec la relation de responsabilité asymétrique. C'est alors qu'Henri Vermorel - qui s'est beaucoup intéressé à Romain Rolland et aux relations de celui-ci avec Freud - me dit: «est-ce que vous avez entendu parler de Laplanche?». Je lui réponds que je connais le Vocabulaire de la psychanalyse: Laplanche et Pontalis. Il me dit: «Laplanche a mis au point toute une théorie sur la relation asymétrique de séduction originaire des parents à l'enfant. Il a parlé de situation anthropologique fondamentale, je vous enverrai son livre.» Quinze jours plus tard, je recevais Les nouveaux fondements pour la psychanalyse et je commençais à lire tout en me disant: «Incroyable!» J'avais trouvé précisément ce qui me manquait, le paradigme que je recherchais: je pouvais combiner l'asymétrie de la séduction originaire et celle de la responsabilité pour l'autre comme quelque chose qui se passait dès le départ de la vie, puis était réactualisé dans la situation analytique. Cela confirmait la pertinence de considérer la situation analytique comme asymétrique, non pas pour des raisons de pouvoir, mais pour ces deux dimensions-là.

ÉB: Comme vous dites, l'asymétrie commence au début de la vie et nous amène aux origines féminines maternelles. J'aimerais bien qu'on en parle du «maternel»... De quel maternel parle-t-on? Et de quel féminin? 
VCV: Dans Autrement qu'être ou au-delà de l'essence, Levinas développe cette idée de responsabilité asymétrique pour l'autre. Il en parle déjà dans les derniers chapitres de son livre Totalité et infini. Il reprendra cela dans son livre suivant alors qu'il décline cette responsabilité pour l'autre en différents termes, ou en différents «non-concepts» comme il les appelle. Il parle de proximité, de vulnérabilité, d'éveil éthique, et aussi de maternité. Une petite note se trouve dans le livre qui s'appelle L'humanisme de l'autre homme; Levinas y rappelle que le mot utérus se dit en hébreu «rehem». Or «rehem» est la racine du terme « rahamim» qui veut dire miséricorde, compassion... On retrouve exactement la même origine en arabe. Et l'islam comme le judaisme font référence à un dieu Rahmān, dieu de miséricorde. Levinas écrit que la responsabilité pour l'autre se rapporte à l'utérus, au maternel, $\mathrm{au}$ «rehem». Il n'en dit pas beaucoup plus, juste une autre petite phrase où il écrit que dans la rencontre à l'autre, je fais de la place dans mes entrailles, je suis éventuellement abîmé dans mes entrailles. Donc il prend cette métaphore du maternel et de l'utérus pour parler de la responsabilité pour l'autre.

Une auteure s'est intéressée à cette question. J'en parle beaucoup dans mon livre, elle s'appelle Beraha Etingger, et elle a interrogé Levinas sur sa position par rapport au maternel quand il parle de responsabilité pour l'autre: il parle de maternel, est-ce qu'il parle d'un maternel également intra-utérin? À partir de là, s'est précisée en moi la relation possible entre cette idée d'accueil, cette idée de réceptivité, de responsabilité pour l'autre - en l'occurrence, mon patient - et le maternel.

Pourquoi parler de féminin-maternel? Parce que chez Levinas, comme chez d'autres, Freud en particulier, se discerne un clivage continu entre le maternel et le féminin: ou il s'agit de maternel, ou l'on s'intéresse au féminin. Le féminin fait référence au sexuel alors que le maternel en serait dissocié. C'est pourquoi j'ai insisté sur le fait que le maternel implique le féminin-maternel.

Ceci dit, j'ai trouvé chez Jacques André un ouvrage, auquel je fais longuement référence, qui s'appelle Aux origines féminines de la sexualité. Jacques André élabore sur le fait que l'enfant venant au monde est nécessairement effracté par la rencontre avec l'adulte doué d'une sexualité consciente et d'un inconscient sexuel, par définition. Pour lui, c'est ainsi que le féminin se creuse, chez tout un chacun, qu'il s'agisse d'un garçon ou d'une fille. En vérité, quand j'ai trouvé ce livre, je me suis dit qu'il allait peut-être évoquer la même conception que la mienne. De fait, même s'il ne mentionnait pas la responsabilité, il décrivait la création du féminin dans l'être. Je me suis dit 
que lorsque je parle de responsabilité pour l'autre — d'éthique à mon avis, tel que Levinas la définit: la responsabilité pour l'autre — je fais référence à un féminin-maternel qui est commun à tous, homme ou femme.

Ensuite, j'ai fait un autre pas à travers mon travail d'écriture. Si, au départ, je savais que j'allais parler de l'asymétrie de la responsabilité pour l'autre et de la séduction originaire, de la passion et de l'affect, de l'analyste, etc., j'ai progressivement réalisé que j'étais en train de préciser les prémisses d'un nouveau paradigme qui situerait l'origine de l'éthique (à différencier de la loi) dans le féminin-maternel de chacun. Plus précisément, lorsqu'un enfant vient au monde, il rencontre un environnement parental adulte qui se doit de le prendre en charge, mais qui de ce fait, est déstabilisé face à la dépendance totale de ce petit (ce nouveau-né est tellement dépendant qu'il va falloir qu'il se consacre à lui afin qu'il survive). Il y a donc une violence inhérente à la rencontre avec le tout-petit, à cette déstabilisation de l'environnement adulte.

Dans un deuxième temps, s'est précisée l'idée d'une transformation de cette déstabilisation en position matricielle. Mon hypothèse est que quelque chose de cette violence et de cette déstabilisation passe dans la psyché du tout-petit et y laisse les traces d'un «tiers matriciel». Ces traces vont former la base de la capacité ultérieure, chez cet homme ou cette femme en devenir, d'être un sujet éthique - ce qui ne veut pas dire que tout humain développera cette capacité.

J'ai ainsi essayé de montrer qu'il y a des prémisses à ce que, par exemple, Melanie Klein a pu exprimer par la prise en compte de l'autre dans la position dépressive. Winnicott a également évoqué le développement de cette aptitude chez l'enfant: vers l'âge de deux ans, quand la mère résiste aux attaques de l'enfant et prend sur elle sa culpabilité potentielle, quelque chose se transforme dans celui-ci qui fonde l'origine de la capacité d'éthique, la capacité morale ou de «concern ». Mon idée est que cette origine est encore plus précoce, à situer au moment même de la toute première rencontre avec l'adulte. L'adulte est à la fois séducteur et émetteur de messages énigmatiques, mais il est aussi émetteur de messages que j’ai appelés "énigm-éthiques», messages qui sont l'effet même de la violence de cette rencontre première. Ceci doit ensuite être élaboré (puisque c'est le fait d'une expérience très précoce), mais je pense qu'il est nécessaire de considérer cette origine.

Il est aussi important de préciser que je ne suis pas du tout d'accord avec l'idée d'une capacité maternelle de dévotion première, qui serait de type instinctuel essentialiste, et à laquelle on revient constamment. Laplanche 
lui-même a avancé cela, et je l'ai repris à mon compte: ce qui témoigne de l'instinct chez la plupart des animaux - la reproduction, ou pour une chatte, de s'occuper de ses petits pendant un mois pour ensuite les abandonner - , est beaucoup plus complexe chez l'humain. Mettre au monde des enfants passe par le désir, et nous savons quels détours peuvent s'y immiscer. De même, je pense qu'il y a quelque chose de fondamentalement humain dans la capacité de responsabilité par rapport à un enfant qui n'a rien à voir avec le niveau instinctuel, même s'il est vrai que pendant deux ou trois semaines après l'accouchement, les effets de l'ocytocine adoucissent (chez certaines mères, mais chez d'autres pas du tout) l'effet de la rencontre et permettent une prise en charge affectueuse et dévouée. Ainsi, une relève doit être assurée, inhérente à l'humanité de cette même femme, du père et de tous les adultes qui sont aux alentours.

ÉB: Ce n'est pas simple, la cette capacité de responsabilité pour l'autre, une capacité maternelle. Cela me fait penser à Liliane Abensour qui parle du maternel sauvage et civilisé; qu'est-ce qui advient de cette autre part du maternel dans cette perspective de capacité de responsabilité pour l'autre?

VCV: Lorsque j'affirme que dans la rencontre avec le petit, ainsi que dans la rencontre avec l'analysant, s'inscrit une forme de violence, je fais aussi allusion à ce qui, dans cette rencontre, peut provoquer chez l'humain, chez l'adulte, un désir de rejet très fort, une haine potentielle, un désir de tuer l'enfant. On voit cela en situation psychotique et chez des femmes qui ont des angoisses infanticides après l'accouchement. Toute cette pulsionnalité existe dans la rencontre première, et quand les choses se passent bien, un travail de transformation permet de la dépasser.

Je ne prétends donc pas que tout est doux et gentil dans cette rencontre... Parfois, la pensée de Levinas est conçue à tort comme une philosophie de la sollicitude, mais il s'agit de quelque chose de beaucoup plus violent. Levinas affirme que dans la rencontre avec l'autre se discerne un «tu ne tueras pas», ce qui indique un dégagement du possible désir de tuer. Cette idée est là, derrière: l'interdit impliqué dans l'idée de responsabilité pour l'autre. Cela pourrait évoquer le maternel Lilithien (ou la femme Lilith). Je rejoins ici une conception de Kristeva: la reliance, et l'éthique de l'érotique maternel. Elle introduit la sublimation, la capacité sublimatrice de la mère qui arrive ainsi à dépasser sa pulsionnalité. Mais Kristeva reste très centrée sur la mère, alors que je m'intéresse à l'origine de la responsabilité pour l'autre, qu'il s'agisse du père ou de la mère. Lorsque je la fonde dans le féminin-maternel, n'allez pas croire qu'il ne s'agit que des mamans, pas du tout! 
ÉB : En vous écoutant parler du tiers matriciel, cela m'amène à poser la question du paternel et du tiers paternel. Mais comment situer le paternel par rapport au tiers matriciel?

VCV: Lors du dernier Congrès des psychanalystes de langue française (CPLF), lequel avait pour thème le paternel, il a évidemment été question de Totem et tabou, de Moïse et le monothéisme, et de la question de la transmission. En travaillant ces deux textes, je me suis rendu compte que Freud mettait systématiquement de côté tout ce qui concernait le féminin-maternel. Or on peut trouver dans Totem et tabou une discussion de Freud avec Atkinson - un historien de l'époque, auquel il se réfère. Atkinson prétend que pour que quelque chose puisse se passer, pour que les fils renoncent à tuer le père et pour qu'on entre dans un monde plus organisé et plus civilisé, il faut une intervention maternelle! Il y avait certainement une femme, dit Atkinson, alors que Freud réplique qu'Atkinson n'étant pas psychanalyste, il ne savait pas que ça ne fonctionne pas comme cela!

En 1931, dans La sexualité féminine, Freud écrit qu'il n'est pas question de penser qu'un enfant puisse vouloir dévorer un père. Pourtant, c'est à partir de cette hypothèse que Freud avait conçu en 1912 Totem et tabou: l'enfant ne peut qu'avoir envie de dévorer une mère et si dévoration fantasmatique du père il y a, c'est évidemment un déplacement de ce désir orienté vers la mère. À la même époque, Melanie Klein évoque essentiellement la même idée. Puis, lorsque Freud écrit Moise et le monothéisme en 1939, il semble avoir oublié ce qu'il avait écrit auparavant, et il reprend toute l'histoire de Totem et tabou, incluant donc la dévoration du père.

Après avoir travaillé Totem et tabou, je remarque que dans Moïse et le monothéisme, Freud, qui suppose l'idée d'un premier Moïse tué, puis d'un second, ne fait que très rapidement allusion aux conditions dans lesquelles Moïse est venu au monde. En effet, Freud, qui connaissait très bien la Bible, affirme que souvent, dans l'histoire de la naissance des grands hommes, l'on retrouve cette notion d'être «sorti des eaux». C'est ainsi qu'il justifie son peu d'intérêt pour les origines éventuelles de Moise. Toutefois, si l'on s'attarde à ce qui est écrit dans la Bible, on peut noter que Moïse vient au monde parce que sa sœur Myriam incite les parents à faire des enfants à une époque critique où l'on mettait à mort tous les premiers-nés. Une femme intervient donc pour qu'un enfant vienne au monde. Puis, sa mère, seconde figure féminine, prend Moïse, le met dans son berceau, et le dépose sur le fleuve; le bébé est ensuite récupéré par une troisième femme, la fille du pharaon, qui décide de prendre l'enfant en charge. Le Midrash raconte que pendant 
trois ans, le petit Moïse continuera d'être allaité par sa propre mère; celle-ci sachant d'avance qu'elle aura à se séparer finalement de son enfant... Donc, trois femmes - Myriam la sœur de Moïse, sa mère Yocheved, et la fille du pharaon - permettent la mise au monde et la poursuite de la vie de cet enfant. Freud a complètement gommé tout cet aspect! Néanmoins, on peut voir à travers ces trois figures féminines — du moins, de la part de la mère de Moïse telle qu'elle est présentée dans le Midrash - quelque chose qui est une position de responsabilité totale: elle allaite son enfant en sachant qu'elle ne le possèdera jamais; c'est la fille de pharaon qui va le faire grandir. Je me suis donc questionnée sur cette trace, ce «tiers matriciel» absent chez Freud, alors que nous travaillions la question du paternel.

J'ai proposé que pour envisager un principe paternel, il fallait d'abord passer par un tiers oublié, le tiers matriciel. J'ai essayé de soutenir que pour qu'une position paternelle incarnant la «loi » prenne acte, il fallait un terrain, un terrain matriciel, à l'origine de l'éthique, relevant du féminin-maternel et existant chez tout adulte. Ce qui revient à proposer que le principe paternel constitue un second temps. Je touche ici à la différence entre l'ordre de la loi et celui de l'éthique. Si la loi se rattache à la justice et au droit, l'éthique évoque le dilemme, le questionnement. Freud s'est peu intéressé à l'éthique comme telle, j'en parle dans mon livre. Pour lui, la question de l'éthique allait de soi, dans une perspective narcissique: se considérer mieux que l'on est. Il s'y est peu intéressé parce que selon moi, il était centré sur la notion de culpabilité, et de ce fait, la question de la responsabilité n'a pas trouvé beaucoup de place dans sa réflexion. Introduire la responsabilité comme concept au cœur de notre réflexion psychanalytique me paraît très important. Je reviens à ce que je disais au départ... Françoise Dolto, d'une manière très simple, a écrit: «Il n’y a rien comme la culpabilité pour nous déresponsabiliser». Cette phrase m’a, à l'époque, beaucoup touchée.

ÉB: Dans votre livre vous parlez des nouvelles technologies; vous faites référence à la procréation assistée et aux transformations actuelles de la structure familiale. Comment articulez-vous la question de l'éthique et la responsabilité pour l'autre dans ces nouvelles perspectives?

VCV: Mon hypothèse est que notre monde occidental est traumatisant, traumatisé, et évidemment post-traumatique. Je pense que de près ou de loin, tout ce monde a été touché d'une manière violente par la Shoah. Il s'est passé, pendant toute cette période nazie, des choses qui ne s'étaient jamais passées auparavant, de l'ordre d'un pervers innommable, qui ont secoué l'humanité occidentale. Qu'on le veuille ou non, qu'on le sache ou 
non, qu'on le refoule ou non, il y a quelque chose dans cette technologie, ce côté technocrate, comme une actualisation qui relève du pervers, où il n'y a pas de respect de l'humain. Il y aurait de nos jours une sorte de continuité de ce qui a pu s'exercer à l'époque, alors que le respect du sujet humain avait disparu et qu'a pu s'exercer une pulsionnalité sexuelle de mort sans limite.

Ce qui se passe dans la société actuelle constituerait une mise en acte continue de ces effets pervers. Il y a bien une recherche apparente de communication avec tout un chacun mais y a-t-il vraiment communication? Je crois qu'il y a une peur terrible de la mort. Donc, il faut faire tout ce qu'on peut, il faut utiliser toutes ses ressources, viser le maximum de jouissance possible, sur fond de pulsions sexuelles de mort.

Il y a certainement des choses qui ont évolué, relativement aux pulsions sexuelles de vie. Évolution difficile mais évolution tout de même, comme le statut des femmes par rapport aux hommes; ça évolue lentement mais cela évolue tout de même.

Dans mon livre, je fais un peu allusion aux procréations assistées; je parle aussi des couples homosexuels hommes ou femmes, qui ont des enfants. J'ai pu observer sur la question homosexualité-hétérosexualité qu'aujourd'hui, on conçoit mieux que chacun fonctionne sur une espèce d'échelle: il y a des individus tout à fait hétérosexuels, il y a des individus tout à fait homosexuels, et il y a des gens qui le sont plus ou moins. De ce fait, je pense que ça nous donne quand même la possibilité d'un regard plus ouvert, plus acceptant et plus tolérant. Certes, le fait de concevoir des enfants dans des conditions qui ne sont pas classiques pose question; comment va-t-on les élever? D’où provient ce désir? Et comment ce désir se transforme-t-il en capacité d'élever ses enfants? Je crois que c'est cela l'essentiel de l'histoire. On peut trouver aujourd'hui des couples homosexuels, en Israël notamment, où deux hommes fonctionnent comme parents avec un couple de femmes: ils mettent au monde des enfants, les éduquent, et les élèvent ainsi. Ceux qui arrivent à fonctionner comme cela font des enfants bien équilibrés, des enfants qui reçoivent beaucoup, avec la conscience, chez tous les partenaires, de l'importance pour l'enfant de savoir qu'il a un papa et une maman d'origine. Finalement, ces enfants devenus adultes vont chercher à avoir des relations hétérosexuelles ou homosexuelles, mais pas nécessairement homosexuelles; en général, ils auraient des relations plutôt hétérosexuelles. Si quelque chose de cette séduction originaire se combine à une éthique de responsabilité aimante - et c'est très souvent ce que l'on voit: des couples extrêmement engagés et investis par rapport à leurs enfants — ça donne de 
très bons résultats. Il est aussi essentiel pour ces enfants de savoir que leurs parents forment un couple dont les partenaires s'apprécient et se désirent mutuellement, que le désir sexuel adulte ne passe pas par l'enfant mais bien par les deux membres du couple. Dans ces conditions, les résultats peuvent être tout à fait positifs, et c'est ce que l'on peut observer.

Néanmoins, cela pose question à plusieurs niveaux... Par exemple, chez les homosexuels, les hommes en particulier, il y a une tendance à intégrer une culture qui souvent, normalise une certaine promiscuité. Au bout d'un moment, certains de ces hommes, las de ce mode de vie, en arrivent à y substituer une relation stable avec un seul partenaire et éventuellement, envisagent de fonder une famille. Notre société est en transformation et il semble qu'aujourd'hui, il y ait plus d'homosexualité patente qu'il y en avait auparavant. Je pense que notre travail d'analyste est de soutenir les homosexuels, hommes ou femmes, qui viennent faire un travail de deuil de leur hétérosexualité, parce qu'en général, cette tendance existe aussi. Je pense d'ailleurs qu'on doit faire le même travail avec les hétérosexuels qui viennent en analyse: ils doivent faire leur deuil de leur homosexualité.

ÉB: Je voudrais revenir à la situation analytique. La question de l'écoute affectée et de la présence affectée. Vous utilisez les concepts de Levinas: celui de la caresse et celui du visage. Des concepts pour lesquels la psychanalyse classique nous a mis en garde; il fallait parler de neutralité bienveillante. Quelle est la place de ces concepts? Que diriez-vous à ce sujet?

VCV: D'abord, le terme «visage» utilisé par Levinas est la traduction du mot «panim». «Panim» chez Levinas c'est «panim Elohim»: les visages de Dieu. Puisque Dieu n'a pas de visage, il y a un paradoxe inhérent à cette idée de visage. «Panim Elohim» c'est le fait que Dieu fait face, il fait face à Moïse mais il n'a pas de visage donc, c'est un visage sans visage. Le visage de Levinas, ce n'est ni les yeux, ni le nez, ni la bouche, ni l'allure du visage, pas du tout. On peut parler de l'interpellation du visage de l'autre alors que la rencontre est avec le dos de l'autre, l'extériorité de l'autre. Le visage c'est ce qui, dans l'être humain, a quelque chose de transcendantal. Nous allons revenir là-dessus.

Quant à la caresse, Levinas utilise ce terme pour décrire une approche de l'autre qui est en douceur, dans une présence qui n'est jamais emprise, qui n'est pas dans le «main-tenant», qui est dans une proximité à partir d'une position de vulnérabilité. La douceur n'est pas le toucher, elle se relie plutôt à la consolation. La caresse ne permet pas à la douleur de s'effacer, il ne s'agit pas de réparer mais d'être avec, et de permettre une certaine ouverture, une 
certaine fenêtre. En effet, nous, analystes, savons qu'en situation de douleur violente, il n'y a plus de futur: tout est dans le présent, un présent qui n'en finirait jamais. Alors que l'idée de caresse témoigne d'un début, d'une fin, d'un temps; elle rétablit une temporalité à travers la présence de l'autre, elle permet une ouverture sur autre chose. Il y a toutefois chez Levinas une certaine ambiguïté dans les termes qu'il utilise. Par exemple, dans Totalité infinie, il parle de la femme, de la maison... et de la caresse en particulier. Il s'en dégage l'idée d'une sexualité infantile, évocatrice pour nous, analystes, mais le sexuel n'est pas ce qui intéresse Levinas à priori. Je pense pourtant qu'il y a quelque chose qu'il fait passer dans les termes qu'il utilise, et qui pour nous fait écho au sexuel infantile et à la séduction originaire.

Laplanche a établi une distinction très claire en affirmant que la seule séduction inhérente à la situation analytique est une réactualisation de la séduction originaire; toute autre séduction représenterait une perversion de la pratique. Du reste, il ne faut pas confondre la séduction originaire avec la séduction que Laplanche qualifie de précoce - soit le fait que les parents prennent leurs petits, les caressent, les embrassent, et leur apprennent ainsi à aimer — qui ne relève évidemment pas de l'analyse! La seule séduction inhérente à l'analyse est l'effet de cette énigme constituée par le refusement de l'analyste - refus de donner des conseils, de répondre à des questions personnelles, etc. - et l'énigme que représente l'offre même d'analyse. Ainsi, la situation analytique et l'analyste qui en fait intégralement partie, incitent le transfert de séduction originaire. Cette séduction peut être particulièrement ressentie lorsque le cadre est déstabilisé. Je donne l'exemple, dans mon livre, d'une patiente que j'avais en analyse à l'époque où l'on avait fait repeindre les portes de mon cabinet. Celle-ci perçut soudain une odeur étrangère, ce qui suscita chez elle des associations douloureuses en rapport avec des souvenirs très premiers: soudain, le «setting» n'était plus le même. Ce changement déclencha un transfert de séduction traumatique que l'on put élaborer. Il peut en être de même dans les moments de séparation; par exemple lorsque l'analyste part en vacances, est malade ou toute autre situation similaire. Des éléments relatifs à la séduction et à l'énigme sont susceptibles de ressortir alors de façon intense. Par exemple, même si l'analyste explique «je ne vais pas vous voir demain, je vous verrai dans deux jours ou bien je vous propose une séance à la place», pour l'analysant, une séance à la place ça n'existe pas comme tel. Ainsi, plusieurs questions peuvent soudain émerger chez lui ou chez elle: "Pourquoi m’a-t-il donné cette séance?», "Pourquoi ne me l'a-t-il pas donnée?», «Qu'est-ce qu'il veut de moi?», «Qu'est-ce qu’il 
cherche?». Ou même: «Pourquoi veut-il être mon analyste? Qu'est-ce qu'il y trouve?», «Il fait ça pourquoi? Il m'aime? Non! Ce n'est pas possible. C'est pour l'argent?», etc. Ces questions sont toujours là même si elles ne sont pas formulées, et elles constituent un moteur, continuellement.

ÉB: Un moteur, et en même temps, comment en rester là? Vous parlez du «makom», du lieu. Le tiers matriciel est comme un lieu; rester là pour écouter de façon affectée cette question: «Qu'est-ce qu'il veut de moi?».

VCV: «Makom», en hébreu, c'est le lieu mais aussi le temps. Lorsque l'on parle de Dieu, on parle du "makom», un lieu-temps qui n'est pas un temps. Le nom de Dieu, en hébreu, c'est à la fois le passé, le présent, et le futur. Ce qu'on traduit en français par Yahvé veut dire, en fait: celui qui a été, celui qui est, et celui qui sera. C'est très intéressant cette espèce de condensation du temps. Dominique Scarfone, dans son rapport ${ }^{3}$, pose une question sur la temporalité ou l'intemporalité dans l'inconscient; il parle de l'impassé. Si Freud a affirmé qu'il n'y a pas de temporalité dans l'inconscient, peut-être est-il plus juste de dire que dans l'inconscient se combinent passé, présent et futur. Ce n'est pas qu'il n'y a pas de temps, mais c'est un temps autre. Étrangement, le concept de Dieu dans le judaïsme correspondrait bien à «l'inconscient», le lieu de cette temporalité intemporelle. Le «makom» analytique serait un lieu, espace et temps, où peut être approché l'inconscient.

Il y a pour moi, dans la rencontre avec l'autre, notre patient, une certaine transcendance, au sens où l'on rencontre un humain, semblable et étranger à la fois, qui nous interpelle d'emblée dans sa fragilité comme dans sa hauteur. Encore une fois, on peut y voir l'idée de Dieu évoquée par la rencontre avec le visage de l'autre, et pour moi, l'idée d'un «makom», lieu analytique qui serait un lieu/temps traversé, imbu de cette transcendance.

J'ai commencé à introduire cette idée de «makom» analytique, et en principe, je devrais la développer plus avant dans un rapport pour le CPLF, un jour, en Israël.

ÉB : C'est très intéressant. Vous nous donnez beaucoup à penser, en particulier sur le processus psychanalytique. D'ailleurs, vous disiez que vous croyiez qu'il n'y avait plus rien à dire là-dessus, mais au contraire! On n'a que peu élaboré, notamment, la question de l'affect. Vous dites d'ailleurs que c'est là le ferment de la psychanalyse: l'écoute affectée, la parole affectée. Pour moi, c'est fondamental ce que vous nous apportez. Il y a bien d'autres choses que l'on pourrait aborder... par exemple, la question de la transmission...

VCV: Oui, la question de la transmission qui traditionnellement (par exemple, dans le judaïsme) passerait par l'homme, par le père, alors que 
je pense que la transmission passe tout autant par le père que par la mère, compte tenu des identifications. Comme dans l'exemple de mon propre père, où j'évoquais l'identification à sa mère; il a transmis quelque chose du maternel qu'il avait complètement internalisé. Je pense que la transmission n'est pas liée au sexe ou au genre: elle est liée à l'emprise affective des personnes qui sont signifiantes chez l'humain depuis sa tendre enfance et tout au long de sa vie.

ÉB: Vous proposez une image qui me parle beaucoup: hélicoïde, vous dites. VCV: Je me suis dit qu'on parle de lignée, ce qui évoque le linéaire. Si je parle en termes d'hélicoïde, cela fait penser à l'ADN, mais ce n'est qu'une métaphore!

ÉB: Tout à fait... Intéressante métaphore qui ramène le paternel et le maternel...

VCV :... en spirale dans la transmission qui influence autant les garçons que les filles, de génération en génération.

Peut-être que je peux vous dire encore un tout petit mot sur mon article, intitulé Edipe meurtri, qui porte sur le trauma et l'Edipe. Plus précisément, dans quels cas l'organisation oedipienne est-elle structurante, et dans quels cas elle peut s'avérer déstructurante. Ce sont les hypothèses de Thierry Bokanowski: le lien avec les éléments traumatiques ou non-traumatiques du début de la vie, qui font que par la suite, l'organisation oedipienne va s'avérer structurante ou non. Lors de cette discussion avec Thierry Bokanowski, comme j'étais interpellée par les anamnèses et les débuts de vie, je me suis intéressée à l'anamnèse d'CEdipe. Or, CEdipe, tout le monde connaît son histoire: il est le fils de Laïos et de Jocaste, sauvé grâce à un serviteur qui, au lieu de le mettre à mort, le sauve et le confie au serviteur du couple royal de Corinthe, ce couple en mal d'enfant et heureux de pouvoir l'adopter. Je me suis dit qu'on retrouvait là une représentation à trois volets combinés de ce qui existe chez tout parent. Le premier volet est le niveau pulsionnel: Laïos veut tuer son fils, donc le premier à avoir envie de tuer ne serait pas l'enfant mais bien le père. Ensuite, il y a Jocaste, la mère qui désire son fils, désir incestueux s'il en est. On retrouve ainsi un désir incestueux et une pulsionnalité agressive mélangés, généralement refoulés, qui trouvent leur origine dans la psyché des parents, mais qui sont transmis aux enfants à titre de messages énigmatiques. C'est le premier volet. Le deuxième volet est représenté par les deux serviteurs, les passeurs: la responsabilité pour l'autre les empêche de tuer l'enfant et les oblige à le garder en vie. Enfin, le couple royal, les parents adoptifs, représentent le troisième volet: celui qui se réfère 
à un narcissisme de vie de bon aloi dès lors qu'ils désiraient un enfant. $\mathrm{Ne}$ pouvant pas le mettre au monde, ils adoptèrent CEdipe et le firent grandir en tout amour et responsabilité. Ces trois volets ne se combinent-ils pas «en bonne santé» chez tout parent, père ou mère?

ÉB: C'est tout à fait intéressant. Le passeur d'ailleurs, on voit très bien le tiers matriciel opérer. Plutôt que de le mener à la mort...

VCV : ... il l'amène à la vie!

ÉB: On transforme tout ce qui est pulsionnel de mort du côté de la vie.

VCV: Absolument. Ce qui n'empêche pas Edipe de terminer comme il a terminé. La tragédie le raconte... C’est peut-être ce qui différencie ce qui reste à un niveau purement fantasmatique chez les parents, de ce qui dans la tragédie de Sophocle se passe vraiment: cette tragédie constituerait un exemple de ce que peut engendrer un trauma effectif, considérant que l'Edipe de Sophocle n'a pas pu traverser son propre Oedipe!

ÉB: Docteure Chétrit-Vatine c'était vraiment un grand plaisir.

VCV: Pour moi aussi, merci beaucoup!

ÉB: J'ai l'impression que vous avez plusieurs pistes de réflexion sur le métier, et c'est avec grand intérêt et beaucoup de plaisir que nous allons vous suivre dans votre travail. Merci beaucoup!

VCV: Merci beaucoup!

\section{Notes}

1. Cette entrevue - également disponible en ligne, sur notre site web - a été réalisée le 29 mai 2014 à Montréal, madame Chetrit-Vatine et monsieur Battat participant l'un et l'autre au Congrès des psychanalystes de langue française.

2. Chetrit-Vatine, V. (2014). The Ethical Seduction of the Analytic Situation, the Feminine Maternal Origins of Responsibility Towards the Other (preface by Dominique Scarfone). London: Karnac/IPA publications.

3. Au Congrès des psychanalystes de langue française, en 2014, à Montréal. 